PROCEEDINGS OF THE

AMERICAN MATHEMATICAL SOCIETY

Volume 137, Number 3, March 2009, Pages 863-867

S 0002-9939(08)09613-5

Article electronically published on September 15, 2008

\title{
PROJECTIVE NORMALITY OF FINITE GROUP QUOTIENTS
}

\author{
S. S. KANNAN, S. K. PATTANAYAK, AND PRANAB SARDAR
}

(Communicated by Ted Chinburg)

\begin{abstract}
In this paper, we prove that for any finite dimensional vector space $V$ over an algebraically closed field $k$, and for any finite subgroup $G$ of $G L(V)$ which is either solvable or is generated by pseudo reflections such that $|G|$ is a unit in $k$, the projective variety $\mathbb{P}(V) / G$ is projectively normal with respect to the descent of $\mathcal{O}(1)^{\otimes|G|}$
\end{abstract}

\section{INTRODUCTION}

Let $G$ be a finite group. Let $V$ be a finite dimensional representation of $G$ over a field $k$. In 1916, E. Noether proved that if the characterstic of $k$ does not divide $|G|$, then the $k$-algebra of invariants $k[V]^{G}$ is finitely generated (see 8 ). In 1926, she proved that the same result holds in all characteristics (see 9]). So, when $k$ is algebraically closed, it is an interesting problem to study GIT-quotient varieties $V / G=\operatorname{Spec}\left(k[V]^{G}\right)$ and $\mathbb{P}(V) / G$ (see [6] and [7]). Also, for every point $x$ in $\mathbb{P}(V)$, the isotropy $G_{x}$ acts trivially on the fiber of the line bundle $\mathcal{O}(1)^{\otimes|G|}$ over $x$. Hence, by Proposition (4.2), page 83 of [5], when $|G|$ is a unit in $k$, the line bundle $\mathcal{O}(1)^{\otimes|G|}$ descends to the quotient $\mathbb{P}(V) / G$, where $\mathcal{O}(1)$ denotes the ample generator of the Picard group of $\mathbb{P}(V)$. Let us denote it by $\mathcal{L}$. On the other hand, $V / G$ is normal. So, it is a natural question to ask if $\mathbb{P}(V) / G$ is projectively normal with respect to the line bundle $\mathcal{L}$. More generally, an interesting question is the following.

Let $G$ be a finite group acting on a projectively normal polarized variety $(X, \mathcal{O}(1))$, where $\mathcal{O}(1)$ is a $G$-linearized very ample line bundle making $X \subset \mathbb{P}(V)$ projectively normal and $\mathcal{L} \in \operatorname{Pic}(X / G)$ is the descent of the line bundle $\mathcal{O}(1)^{\otimes|G|}$ on $X$. Is the polarized variety $(X / G, \mathcal{L})$ projectively normal?

In this note, we give an affirmative answer to this question in the following two cases:

(1) $G$ is solvable and the characteristic of the base field does not divide $|G|$;

(2) $G$ is a finite subgroup of $G L(V)$ generated by pseudo reflections, where $V$ is a finite dimensional vector space over a field $k$ of characteristic not dividing $|G|$.

Proof of the main result essentially uses an arithmetic result due to Erdös-GinzburgZiv (see [2]).

Received by the editors July 5, 2007, and, in revised form, March 4, 2008.

2000 Mathematics Subject Classification. Primary 14Lxx.

Key words and phrases. Pseudo reflections, line bundle.

(C)2008 American Mathematical Society 


\section{Solvable CASE}

In this section, we prove the following proposition, which can be applied to prove our main result when the group $G$ is solvable.

Proposition 1.1. Let $G$ be a finite solvable group, and let $V$ be a finite dimensional faithful representation of $G$ over a field $k$ of characteristic not dividing $|G|$. Let $m=|G|, R:=\bigoplus_{d \geq 0} R_{d} ; R_{d}:=\left(S y m^{d m} V\right)^{G}$. Then $R$ is generated as a $k$-algebra by $R_{1}$.

Proof. Step 1: We first prove the statement when $G$ is cyclic of order $m$. Let $\xi$ be a primitive $m$-th root of unity in an algebraic closure $\bar{k}$ of $k$. Let $F=k(\xi)$. Since $F$ is a free $k$-module, we have $V^{G} \otimes_{k} F=\left(V \otimes_{k} F\right)^{G}$. Hence, we may assume that $\xi \in k$.

Let $G=\langle g\rangle$. Write $V=\bigoplus_{i=0}^{m-1} V_{i}$ where $V_{i}:=\left\{v \in V: g \cdot v=\xi^{i} \cdot v\right\}$, $0 \leq i \leq m-1$. Now, let $f \in R_{d}$ be of the form $f=X_{0} \cdot X_{1} \cdots X_{m-1}$ with $X_{i} \in \operatorname{Sym}^{a_{i}} V_{i}$ such that $\sum_{i=0}^{m-1} a_{i}=d m$. Since $f$ is $G$-invariant we have

$$
\sum_{i=0}^{m-1} i . a_{i} \equiv 0 \bmod m \text {. }
$$

If $d=1, f \in R_{1}$, so we may assume that $d \geq 2$. Now, consider the sequence of integers

$$
\underbrace{0, \ldots, 0}_{a_{0} \text { times }}, \underbrace{1, \ldots, 1}_{a_{1} \text { times }}, \cdots, \underbrace{m-1, \ldots, m-1}_{a_{m-1} \text { times }} .
$$

Since the sequence has $d m$ terms and $d \geq 2$, by a theorem of Erdös-Ginzburg-Ziv (see [2]), there is a subsequence with exactly $m$ terms whose terms add up to a multiple of $m$. Thus there exist $f_{1} \in R_{1}$ and $f_{2} \in R_{d-1}$ such that $f=f_{1} \cdot f_{2}$. Hence the proof follows by induction on $\operatorname{deg}(f)$.

Step 2: Now we assume that $G$ is any finite solvable group of order $m$. We use induction on $m$ to prove the statement. We may assume that $m$ is not a prime number. Since $G$ is solvable it has a normal subgroup $H$ such that $G / H$ is a cyclic group of prime order.

Let $W:=\left(S y m^{|H|} V\right)^{H}$. Since $H$ is a normal subgroup of $G$, both $\underbrace{W \otimes \ldots \otimes W}_{d|G / H| \text { copies }}$

and $\left(S y m^{d|H|} V\right)^{H}$ have natural $G / H$-module structures. Let $G_{1}=G / H$. Since $|H|<|G|$, by induction, the homomorphism $\underbrace{W \otimes \ldots \otimes W}_{d\left|G_{1}\right| \text { copies }} \longrightarrow\left(S y m^{d|G|} V\right)^{H}$ is surjective.

Claim. The natural map $\left(S y m^{d\left|G_{1}\right|} W\right)^{G_{1}} \longrightarrow\left(S y m^{d|G|} V\right)^{G}$ is surjective.

The surjectivity of the natural map $S y m^{d .\left|G_{1}\right|} W \longrightarrow\left(S y m^{d|G|} V\right)^{H}$ of $G_{1}$-modules follows from (1) and the following commutative diagram:

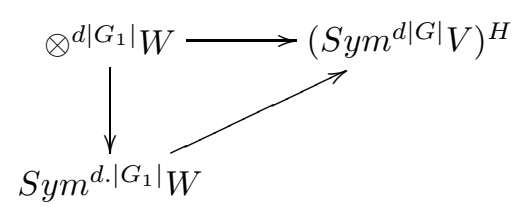

Hence applying Reynold's operator we have the claim. 
Now, consider the commutative diagram:

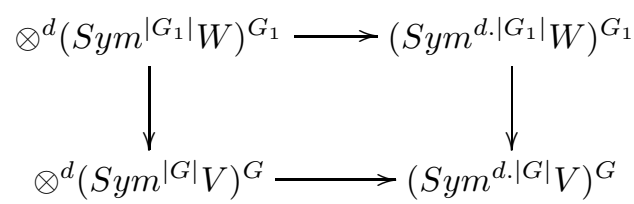

The first horizontal map is surjective by step (1) and the second vertical map is surjective by (2). Thus the second horizontal map is surjective. Thus the proposition follows.

\section{Group generated By PSEUdo REFleCtions}

In this section, we will prove a combinatorial lemma which can be applied to prove our main result when the group $G$ is generated by pseudo reflections.

Let $\underline{a}=\left(a_{1}, a_{2}, \cdots, a_{r}\right) \in \mathbb{N}^{r}$ and $N_{\underline{a}}=\prod_{j=1}^{r} a_{j}$. Consider the semigroup

$$
M_{\underline{a}}=\left\{\left(m_{1}, m_{2}, \cdots, m_{r}\right) \in \mathbb{Z}_{\geq 0}^{r}: \sum_{j=1}^{r} m_{j} a_{j} \equiv 0 \bmod N_{\underline{a}}\right\}
$$

and the set

$$
S_{\underline{a}}=\left\{\left(m_{1}, m_{2}, \cdots, m_{r}\right) \in \mathbb{Z}_{\geq 0}^{r}: \sum_{j=1}^{r} m_{j} a_{j}=N_{\underline{a}}\right\} .
$$

Lemma 2.1. $M_{\underline{a}}$ is generated by $S_{\underline{a}}$ for $\underline{a} \in \mathbb{N}^{r}$.

Proof. Suppose $\left(m_{1}, m_{2}, \cdots, m_{r}\right) \in \mathbb{Z}_{\geq 0}^{r}$ such that

$$
\sum_{j=1}^{r} m_{j} \cdot a_{j}=q \cdot N_{\underline{a}}, \text { with } q \geq 2 .
$$

Let $n=q \cdot N_{\underline{a}}$. Choose any $n \times r$ matrix

$$
A=\left(\begin{array}{cccc}
x_{11} & x_{12} & \cdots & x_{1 r} \\
x_{21} & x_{22} & \cdots & x_{2 r} \\
\vdots & \vdots & \ddots & \vdots \\
x_{n 1} & x_{n 2} & \cdots & x_{n r}
\end{array}\right)
$$

with entries in $\{0,1\}$ such that each row sum $\sum_{j=1}^{r} x_{i, j}$ is equal to 1 and for each $j=1,2, \cdots, r$, the $j$ th column sum $\sum_{i=1}^{n} x_{i, j}$ is $m_{j} a_{j}$.

Since $q \geq 2, n \geq 2 . a_{1}$, the sequence $\left\{x_{11}, x_{21}, \cdots, x_{n 1}\right\}$ has at least $2 a_{1}$ terms. Hence, applying the theorem of Erdös-Ginzburg-Ziv repeatedly (see 2]) and rearranging the entries of the first column if necessary, we can assume that the $n$ terms of the sequence can be partitioned into $\frac{n}{a_{1} a_{2}}$ subsequences $\left\{x_{11}, x_{21}, \cdots, x_{a_{1} 1}\right\}$, $\left\{x_{\left(a_{1}+1\right) 1}, x_{\left(a_{1}+2\right) 1}, \cdots, x_{2 a_{1} 1}\right\}, \cdots,\left\{x_{\left(n-a_{1}+1\right) 1}, x_{\left(n-a_{1}+2\right) 1}, \cdots, x_{n 1}\right\}$, each of length $a_{1}$, and the sum of the terms of each of these subsequences is a multiple of $a_{1}$.

Again, consider the sequence $\left\{\sum_{i=1}^{a_{1}} x_{i 2}, \sum_{i=a_{1}+1}^{2 a_{1}} x_{i 2}, \cdots, \sum_{i=n-a_{1}+1}^{n} x_{i 2}\right\}$. Using the same argument as above we can assume that this sequence can be partitioned into $\frac{n}{a_{1} a_{2}}$ subsequences, each of length $a_{2}$, and the sum of the terms of each subsequence is a multiple of $a_{2}$.

Proceeding in this way, we can see that for each $j=1,2, \cdots, r$, the sum of the first $N_{\underline{a}}$-terms in the $j$ th column of the matrix $A$ is a multiple of $a_{j}$. 
Let $b_{j}=\sum_{i=1}^{N_{a}} x_{i j}$. By construction of the $x_{i j}$ 's,$b_{j}$ is a multiple of $a_{j}$ for every $j=1,2, \cdots, r$. So, for each $j=1,2, \cdots, r$, write $b_{j}=a_{j} c_{j}$, with $c_{j} \in Z_{\geq 0}$. Now, since $\sum_{j=1}^{r} x_{i, j}=1$, for each $i=1,2, \cdots, n$, we have $\sum_{j=1}^{r} b_{j}=\bar{N}_{\underline{a}}$. Hence, $\left(c_{1}, c_{2}, \cdots, c_{r}\right) \in S_{\underline{a}}$. As $m_{j} a_{j}=\sum_{i=1}^{n} x_{i j}$, we have $b_{j} \leq m_{j}$ for every $j=$ $1,2, \cdots, r$. Thus, we have $\left(m_{1}, m_{2}, \cdots, m_{r}\right)=\left(c_{1}, c_{2}, \cdots, c_{r}\right)+$ $\left(m_{1}-c_{1}, m_{2}-c_{2}, \cdots, m_{r}-c_{r}\right)$, with $\sum_{j=1}^{r}\left(m_{j}-c_{j}\right) a_{j}=(q-1) N_{\underline{a}}$. So, the lemma follows by induction on $q$.

Corollary 2.2. Let $V$ be a finite dimensional vector space over a field $k$. Let $G$ be a finite subgroup of $G L(V)$ which is generated by pseudo reflections. Further assume that the characteristic of $k$ does not divide $|G|$. Then $R=\bigoplus_{d \in \mathbb{Z}_{\geq 0}} R_{d}$, where $R_{d}:=\left(\text { Sym }^{d .|G|}\left(V^{*}\right)\right)^{G}$, is generated by $R_{1}$.

Proof. By a theorem of Chevalley-Serre-Shephard-Todd (see [1, 4, 10], 11]), $\left(\operatorname{Sym}\left(V^{*}\right)\right)^{G}$ is a polynomial ring $K\left[f_{1}, f_{2}, \cdots, f_{r}\right]$ where each $f_{i}$ is a homogeneous polynomial of degree $a_{i}$ and $\prod_{i=1}^{r} a_{i}=|G|$. Thus, the proof follows from Lemma 2.1.

\section{MAIN THEOREM}

We now prove our main result.

Let $G$ be a finite group and $V$ be a finite dimensional, faithful representation of $G$ over an algebraically closed field of characteristic not dividing $|G|$. Let $\mathcal{O}(1)$ denote the ample generator of the Picard group of $\mathbb{P}(V)$. Let $\mathcal{L}$ denote the descent of the line bundle $\mathcal{O}(1)^{\otimes|G|}$ to the quotient $\mathbb{P}(V) / G$.

Before stating the main result, we recall the following.

1. A polarized variety $(X, \mathcal{L})$ where $\mathcal{L}$ is a very ample line bundle is said to be projectively normal if its homogeneous coordinate ring $\bigoplus_{n \in \mathbb{Z} \geq 0}\left(H^{0}\left(X, \mathcal{L}^{\otimes n|G|}\right)\right)$ is integrally closed. For a reference, see exercise 3.18, page 23 of [3].

2. The polarized variety $(\mathbb{P}(V) / G, \mathcal{L})$ is

$$
\operatorname{Proj}\left(\bigoplus_{n \in \mathbb{Z}_{\geq 0}}\left(H^{0}\left(\mathbb{P}(V), \mathcal{O}(1)^{\otimes n|G|}\right)\right)^{G}\right)=\operatorname{Proj}\left(\bigoplus_{n \in \mathbb{Z}_{\geq 0}}\left(\operatorname{Sym}^{n|G|}\left(V^{*}\right)\right)^{G}\right) .
$$

For a reference, see Theorem 3.14 and page 76 of [6].

Now, let us assume that either $G$ is solvable or is generated by pseudo reflections in $G L(V)$. Then, we have

Theorem 3.1. $\mathbb{P}(V) / G$ is projectively normal with respect to $\mathcal{L}$.

Proof. The proof follows from Proposition 1.1, Corollary 2.2, and exercise 5.14(d), Chapter II of [3].

Remark. It is an interesting and important problem to answer the following question.

Let $G$ be a reductive group (not necessarily finite) acting morphically on a projective variety $X$. Let $\mathcal{M}$ be a $G$-linearized very ample line bundle on $X$ such that $\mathcal{M}$ descends to the quotient $X / / G$. Let $\mathcal{L}$ be the descent. Is the polarized variety $(X / / G, \mathcal{L})$ projectively normal?

\section{ACKNOWLEDGEMENT}

We thank the referee for valuable comments and suggestions. 


\section{REFERENCES}

[1] C. Chevalley, Invariants of Finite Groups Generated by Reflections, Amer. J. Math. 77 (1955), 778-782. MR0072877 (17:345d)

[2] P. Erdös, A. Ginzburg, and A. Ziv, A theorem in additive number theory, Bull. Res. Council, Israel, $10 \mathrm{~F}$ (1961), 41-43.

[3] R. Hartshorne, Algebraic Geometry, Graduate Texts in Math., 52, Springer-Verlag, New York-Heidelberg, 1977. MR0463157 (57:3116)

[4] R. Kane, Reflection Groups and Invariant Theory, CMS Books in Mathematics, SpringerVerlag, New York, 2001. MR1838580 (2002c:20061)

[5] H. Kraft, P. Slodowy and T. A. Springer, eds., Algebraic Transformation Groups and Invariant Theory, Birkhäuser-Verlag, Basel, 1989. MR1044582 (91m:14074)

[6] D. Mumford, J. Fogarty and F. Kirwan, Geometric Invariant Theory, Springer-Verlag, Berlin, 1994. MR1304906 (95m:14012)

[7] P. E. Newstead, Introduction to Moduli Problems and Orbit Spaces, TIFR Lecture Notes, Narosa, New Delhi, 1978. MR546290 (81k:14002)

[8] E. Noether, Der Endlichkeitssatz der Invarianten endlicher Gruppen, Math. Ann. 77 (1915), 89-92. MR1511848

[9] E. Noether, Der Endlichkeitssatz der Invarianten endlich linearer Gruppen der Charakteristik $p$, Nachr. Akad. Wiss. Gottingen (1926), 28-35.

[10] J-P. Serre, Groupes finis d'automorphismes d'anneaux locaux réguliers, Colloq. d'Alg. École Norm. de Jeunes Filles, Paris (1967), 1-11.

[11] G. C. Shephard and J. A. Todd, Finite Unitary Reflection Groups, Canadian J. Math. 6 (1954), 274-304. MR0059914 (15:600b)

Chennai Mathematical Institute, Plot H1, Sipcot it Park, Padur Post Office, Siruseri, Tamilnadu, 603103, India

E-mail address: kannan@cmi.ac.in

Chennai Mathematical Institute, Plot H1, Sipcot it Park, Padur Post Office, Siruseri, Tamilnadu, 603103, India

E-mail address: santosh@cmi.ac.in

Chennai Mathematical Institute, Plot H1, Sipcot it Park, Padur Post Office, Siruseri, Tamilnadu, 603103, India

E-mail address: pranab@cmi.ac.in 